\title{
Sistema Integrado de Evaluación Diferenciada para Adolescentes y Jóvenes, SIED-AJ, desde el quehacer de programas de ejecución de sanciones de Corporación Opción
}

\author{
Integrated System for Differential Assessment of \\ Adolescents and Youth, SIED-AJ, from the perspective of \\ Corporation Opción's sanction implementation programs
}

Juan Pablo Cortés ManríQuez. Psicólogo, mágíster director Programa de Libertad Asistida Especial El Bosque, Corporación Opción.jpcortes@opcion.cl

Christian Lorca Pastene. Psicólogo, director Programas de Libertad Asistida y Libertad Asistida Especial Maipú, Corporación Opción.clorca@opcion.cl.

Osvaldo VázQuez Rossoni. Psicólogo, coordinador nacional Programas de Justicia Penal Adolescente, Corporación Opción. ovazquez@opcion.cl

\begin{abstract}
Resumen
El Sistema Integrado de Evaluación Diferenciada para Adolescentes y Jóvenes, SIED-AJ, es un instrumento para el diagnóstico, cuyo objetivo se centra en el reconocimiento del adolescente o joven que comete una infracción de la ley como sujeto de derechos, que se encuentra en una etapa de desarrollo especifica, y en donde se reconozcan sus recursos, potencialidades y factores de riesgo (o vulnerabilidad), con el propósito de identificar niveles de complejidad y así orientar intervenciones diferenciadas, especificas, focalizadas e idóneas de acuerdo a sus necesidades. EI SIED-AJ es una herramienta práctica conformada por la teoría y la evidencia científica, cuenta con ocho variables con sus respectivas dimensiones y categorías, cuya finalidad es generar un tipo de análisis retrospectivo, exponiéndose elementos que apoyan la definición de la gestión del proceso de intervención con criterios específicos para cada variable con la finalidad de modificar la ocurrencia de conductas de tipo delictual.
\end{abstract}

Palabras Clave. Enfoque de Derechos, Adolescente, Infractores de Ley, Instrumentos, Niveles de Complejidad.

\footnotetext{
Abstract

The Integrated System of Differentiated Assessment for Adolescents and Youths, (SIED-AJ for its Spanish Acronym) is a diagnostic instrument aimed at recognizing the offending adolescent and/ or youth, as subject of Law who is at a specific development stage and where their resources, potentialities and risk factors (or vulnerability) may be recognized in order to identify levels of complexity and thus advise on differentiated, specific and focused interventions in accordance with their needs.

The SIED-AJ is a practical, theory-and evidence-informed tool; it consists of 8 variables with their applicable dimensions and categories aimed at generating a type of retrospective analysis also exposing elements which define the intervention process management with specific criteria for each variable in order to modify the occurrence of crime-typed behaviours.
}

1 Propiedad Intelectual SIED-AJ: Corporación Opción, www.opcion.cl, Carlos Justiniano No 1123; Providencia, Santiago. 


\section{Introducción}

El SIED-AJ tiene por objetivo determinar niveles de complejidad para la intervención, a partir de las necesidades y las situaciones presentadas por los adolescentes y jóvenes entre los 14 y 18 años de edad que ingresan a los programas de ejecución de sanciones de Libertad Asistida (PLA) y Libertad Asistida Especial (PLE) según lo establecido por juzgados de garantía o Juzgado Oral en lo Penal.

Dicha clasificación determina niveles de complejidad que permiten orientar intervenciones diferenciadas, focalizadas, especializadas e idóneas, además de evaluar sistemáticamente niveles de cambio y logros en el proceso psicosocioeducativo. Como instrumento mide las características de los y las adolescentes y jóvenes, según un nivel de complejidad en relación con la capacidad de respuesta que tiene la propuesta de intervención. Genera insumos para redefinir las adecuaciones que son necesarias de incorporar por las situaciones más complejas identificadas en el ingreso. Este dispositivo estructura y cohesiona desde sus inicios la intervención, apoyando la planificación y la permanente supervisión de casos. Además, proporciona, en una segunda medición, ex post, el nivel de logro o cambio de las situaciones evaluadas al final del proceso.

Por tal razón, responde a la necesidad de mejorar la propuesta de intervención, focalizando y adecuando las estrategias acordes a los jóvenes que ingresan, y asegurar que todos tengan una oferta programática de trabajo idónea a las condiciones que presenta el adolescente o joven asegurando que ésta sea coherente a cada situación particular. El SIED-AJ identifica el grupo de jóvenes que según el juicio profesional tienen la necesidad de redefinir o adecuar acciones específicas para el logro de los objetivos. Por otro lado, es durante el proceso de intervención en donde este diseño cobra mayor importancia y funcionalidad a los equipos de intervención directa, en tanto es coadyuvante a la supervisión y seguimiento de casos, consiguiendo objetivar los procesos y generar parámetros que contrastan la consistencia y coherencia de la intervención ejecutada.

\section{Marco referencial}

Los fundamentos en donde se sustenta el SIED-AJ son a partir de los siguientes principios y marcos normativos:

- La Declaración Universal de los Derechos Humanos (1948) establece que todos los seres humanos nacen libres e iguales en dignidad y derechos sin distinción alguna de raza, color, sexo, idioma, religión, opinión política o de cualquier otra índole, origen nacional o social, posición económica, nacimiento o cualquier otra condición. Por lo tanto, desde esta distinción resulta fundamental favorecer prácticas distintivas que prioricen la condición establecida en la Declaración de los DD.HH.: que la infancia tiene derecho a cuidados y asistencia especial.

- La Convención Internacional de Derechos del Niño (CIDN) que en 1989 promovió el cambio de visión en América Latina acerca del respeto y protección de los derechos del niño. A partir de esa instancia se definieron los estándares de protección de los derechos de los adolescentes que tienen conflicto con la ley. En su artículo 40 sostiene que "aquellos niños que hayan infringido las leyes, sean acusados o declarados culpables, deben ser tratados de manera en que se fomente su dignidad, respetando los derechos humanos y libertades fundamentales de terceros". Además, sostiene que se debe tener en cuenta la edad del niño y promover su reintegración y que éste asuma una función constructiva en la sociedad.

- Ley de Responsabilidad Penal Adolescente (LRPA), Ley 20.084, aprobada en el año 2005 y vigente desde junio del año 2007. Ésta se implementa en el marco de la Reforma Procesal Penal, pasando, en Chile, de un sistema de persecución inquisitivo a uno acusatorio y garantista. Desde una nueva perspectiva, la ley está orientada ya no al castigo-retribución, sino que responsabilización del adolescente culpado de un ilícito dentro de un marco de promover la protección de derechos y reinserción social del adolescente (Corporación Opción, UNICEF, 2009). De tal modo, la finalidad de las sanciones que se propone la LRPA (Art. 20) es "hacer efectiva la responsabilidad de los adolescentes por los hechos delictivos que cometan, de tal manera que la sanción forme parte de una intervención socioeducativa amplia y orientada a la plena integración social" (Biblioteca del Congreso Nacional de Chile, 2005).

- Una orientación fundante del SIED-AJ se centra en la justicia restaurativa, que se puede definir como "un proceso a través del cual todas las partes involucradas en un determinado delito participan para resolver de manera colectiva una forma para lidiar con las consecuencias del delito y sus implicaciones para el futuro". (Marshall, 1999).

- Dentro de las perspectivas teóricas, que nutren 
la concepción y desarrollo del SIED-AJ, se puede mencionar el enfoque evolutivo, que permite definir estrategias acordes al desarrollo o ciclo vital en el que se encuentra cada individuo. Se sustenta en los siguientes supuestos: el desarrollo tiene lugar en etapas sucesivas, claramente definidas y consideran una secuencia constante; cada fase del ciclo se caracteriza por sucesos que deben ser resueltos de manera satisfactoria para poder avanzar al siguiente. De no ser así, las etapas posteriores evidenciarán niveles de déficit, pudiendo expresarse en desadaptaciones físicas, cognitivas, emocionales o sociales y; cada fase contiene un rasgo dominante, un complejo de rasgos o punto crítico que la distingue de las fases anteriores o posteriores. (Vázquez, 2003).

- El enfoque de género; éste hace referencia a "la construcción sociocultural de las diferencias sexuales; siendo las diferencias biológicas las que dan pie a los conceptos de masculinidad y femineidad, asi como también a los elementos valorativos en torno a hombres y mujeres, lo cual es fruto de un proceso histórico". (Sename 2011). En el ámbito de la infracción penal adolescente resulta entonces relevante considerar este aspecto del desarrollo evolutivo en el proceso de formación de la identidad de género, en tanto suelen responder a los estereotipos de que van distribuyendo atributos a lo femenino y masculino de manera diferenciada, en tanto parecen encontrarse relacionadas con la comisión de cierto tipo de conductas infractoras como una forma de construir, consolidar la propia identidad adolescente en cuanto masculinidad y femineidad, y responder a la socialización familiar y sociocultural respecto de los modelos construidos (ONU, 2010). Además, las relaciones de género no ocurren en el vacío sino, por el contrario, en el panorama dibujado por la clase, la sexualidad, la edad, y otros factores que conducen a la vulnerabilidad y a la desigualdad. Ello genera una matriz de dominación que no sólo produce relaciones de subordinación sino agenciamientos y formas de resistencia (Daly y Stephens 1995).

- El enfoque socioeducativo plantea una mirada integradora para trabajar con adolescentes o jóvenes en situación de conductas infraccionales y desde esta perspectiva acompañar los procesos de responsabilización desde una mirada socioeducativa. De este modo, se postula la educación popular como metodología de trabajo en contextos de socioeducación con adolescentes o jóvenes infractores de la ley, en tanto que las técnicas participativas desde esta línea surgen como herramientas educativas que buscan provocar la participación para la reflexión y el análisis (Bustillos, 2001). Tales técnicas intentan recoger lo subjetivo y objetivo de la práctica y el contexto en que se mueven los sujetos para promover una reflexión educativa de ello (Bustillos, 2001).

- El modelo de Riesgo-Necesidad-Respuesta (RNR), que es quizás el modelo más influyente para la evaluación y tratamiento de adolescentes que cometen conductas infraccionales (Blanchette y Brown, 2006; Ward, Mesler y Yates, 2007). El modelo RNR se ha elaborado y contextualizado dentro de una teoría general de la personalidad y la teoría cognitiva del aprendizaje social de la conducta criminal (Andrews y Bonta, 2006). Desde 1990, una serie de principios se ha añadido a los principios teóricos básicos para mejorar y fortalecer el diseño e implementación de intervenciones efectivas. Los tres principios básicos son (Bonta y Andrews, 2006):

Principio de Riesgo: Parear el nivel de intensidad de la intervención con el riesgo de reincidencia.

Principio de Necesidad: Evaluar las necesidades criminógenas para que estas orienten el tratamiento.

Principio de Respuesta: Maximizar la capacidad del adolescente para aprender de una intervención de rehabilitación mediante un tratamiento cognitivo-conductual y la adaptación de la intervención al estilo de aprendizaje, motivación, habilidades y puntos fuertes (recursos) del adolescente.

- El paradigma del desistimiento, orientación teórica en que se funda el SIED-AJ, plantea que existe un proceso de abandono esperable de la vida delictual y este va acompañado de una narrativa de cambio de el o la adolescente, por lo tanto, resulta importante conocer los factores que influyen en el surgimiento de estas narrativas (Moliné y Olivé, 2011). Desde este enfoque se plantea la importancia de considerar el vínculo del joven con la sociedad a través de su contacto con instituciones sociales como la iglesia, la familia, el trabajo (Laub y Sampson 2003). El desarrollo de una identidad prosocial (Maruna 2001; Paternóster y Bushway, 2009), cambios cognitivos que facilitan modificaciones (Giordano et al., 2002), sentido de agencia o autoeficacia (Shapland y Bottoms, 2011).

- El enfoque ecológico del desarrollo humano 
(Bronfenbrenner, 1979), examina el cambio de la conducta en el individuo a través de su relación con el contexto. El modelo ecológico plantea una visión integral, sistémica y naturalista del desarrollo psicológico entendiéndolo como un proceso complejo, que responde a la influencia de una multiplicidad de factores estrechamente ligados al ambiente o entorno ecológico en el que dicho desarrollo tiene lugar (Torrico y Santin, 2002). Se considera el desarrollo humano como una progresiva acomodación entre un ser humano activo y sus entornos inmediatos. Pero este proceso, además, se ve influido por las relaciones que se establecen entre estos entornos y por contextos de mayor alcance en los que están incluidos esos entornos (Bronfenbrenner, 1979) (Torrico y Santin, 2002).

\section{Metodología evaluación diferenciada (SIED-AJ)}

El SIED-AJ, como diseño de evaluación diferenciada, es una construcción resultado de la revisión de la evidencia científica actualizada recogida a través de los diversos instrumentos, que a nivel nacional e internacional se encuentran validados y que cuentan con reconocimiento a nivel de la comunidad científica. Sin embargo, según sus objetivos, metodologías, gestión de aplicación, tipos de administración y centrados en los factores de riesgo del sujeto observado, no respondían a los requerimientos de los equipos de trabajo de intervención directa, que demandan procedimientos estructurados que orienten la toma de decisión frente a las problemáticas particulares de cada adolescente o joven, con la finalidad de interrumpir trayectorias delictivas y propiciar el desistimiento e integración social de los usuarios de cada programa.

En este sentido, la evaluación de complejidad cambia el centro de observación, desde situar la dificultad en lo complejo de la persona (personaproblema) a comprenderlo desde las posibilidades de gestión de caso que tiene el programa, en cuanto a las acciones y estrategias definidas. Lo innovador del SIED-AJ es haber pasado de instrumentos que sitúan la complejidad y las dificultades en el sujeto a éste, que pone la complejidad en la intervención que debe hacer el profesional y el equipo que interviene, poniendo el centro en visualizar las condiciones y capacidades actuales que tiene el programa para abordarlas.

Este instrumento permite al final de la intervención evaluar resultados en el proceso socioeducativo, estimando indicadores confiables para mejorar la eficacia de las acciones de intervenciones en pos de la inserción social y la promoción del ejercicio ciudadano.

La aplicación se desarrolla con facilidad por la cercanía y naturalidad que presenta al momento de ser incorporada en el quehacer cotidiano del profesional a cargo de la intervención, dado que se utiliza como parámetro de contraste para evaluar la ejecución de su propio proyecto.

Para comprender la complejidad de las situaciones en los adolescentes y jóvenes que cometen conductas infractoras, son relevantes tanto los parámetros individuales como los sociales y culturales. Estos también son expresiones de contextos macrosociales que influyen en cada caso y que se encuentran fuera del ámbito de intervención. En este sentido se propone plantear la complejidad desde el quehacer cotidiano de la experiencia y las capacidades de adaptación por parte de los profesionales, a este tipo de situaciones. Asimismo, se consideran las prácticas de los programas de intervención y las posibilidades que ofrecen en innovación y flexibilidad real para asegurar el cumplimiento dentro de un análisis integral del fenómeno.

El SIED-AJ como instrumento de trabajo no tiene como propósito predecir conductas o comportamiento futuros definidos como niveles de riesgo de reincidencia. Su objetivo es:

- Describir la situación de los adolescentes y jóvenes, entregando criterios homologables de complejidad.

- Ordenar y orientar el proceso de diagnóstico como evaluación inicial y apoyar la elaboración de estrategias idóneas ajustadas al nivel de complejidad que presentan.

- Determinar jerarquías de ámbitos para la intervención y líneas de trabajo con mayor coherencia en sus acciones.

- Fortalecer las herramientas del profesional a cargo del caso, aportando en los aspectos del seguimiento y supervisión del caso en las evaluaciones ex dure, visualizar desde el inicio del proceso las situaciones que permiten posteriormente ser contrastados con resultados para medir niveles de logro al momento de la evaluación ex post.

En síntesis, la utilización de este diseño apoya los procesos de supervisión y seguimiento de casos, generando parámetros que contrastan la consistencia y coherencia de los procesos de intervención 
ejecutados en los programas de justicia juvenil, a partir de los datos que se recogen desde el ingreso hasta el término del diagnóstico, visualizando con ello la necesidad de implementar adecuaciones de estrategias de focalización en los factores que identifica según su nivel de complejidad. Por otro lado, al focalizar y estructurar la intervención implica no sólo medir resultados, sino que también avanzar en el mejoramiento de los estándares de calidad de los programas.

El análisis de resultados se utiliza con el fin de orientar estrategias nuevas para enfrentar situaciones más complejas que enfrenta el adolescente. Asimismo, se busca examinar si las estrategias que se están implementando proveen respuestas satisfactorias desde el programa para ajustar las estrategias durante el proceso de intervención.

\section{Diseño metodológico (SIED-AJ) Características del diseño de evaluación (Retrospectivo - Descriptivo)}

Una de las características principales del diseño es que cuenta con un tipo de análisis retrospectivo. Esto supone el tratamiento sistemático de las variables observadas producidas con anterioridad a la aplicación del diseño. Los datos corresponden a la aplicación (tiempo 0) hacia atrás, siendo el tiempo 0 el momento de llenado de la ficha de ingreso. Esto significa que desde el momento de la aplicación son posibles de reconocer los efectos de causas anteriores, previas y ajenas a la observación. En una segunda aplicación, durante el momento del egreso, ofrecer una alternativa para realizar juicios evaluativos referidos al cambio de las situaciones iniciales y a las estrategias que se aplicaron durante el proceso. El carácter descriptivo busca medir las propiedades o atributos del fenómeno observado; por lo tanto, el diseño permite indagar la incidencia de las variables, analizadas por separado, de manera independiente y con posterioridad integrarlas para dar un panorama general al final de su aplicación, tanto al ingreso como durante y al egreso, generando un dispositivo sistemático de aplicación y descripción (Hernández, Fernández, Baptista; 1998).

\section{Aspectos técnicos}

El SIED-AJ es el resultado de la revisión de evidencia recogida a través diversos marcos teóricos e instrumentos internacionales. Es un dispositivo que cohesiona y estructura la intervención, con base en variables validadas por medio de la evidencia comparada a nivel internacional y que se adaptan a las necesidades contextuales de cada programa del país.

\section{Índices y escala}

La construcción de un índice ofrece un manejo sencillo para su gestión y análisis dado que reduce las variables a una medición cuantitativa de fácil comprensión, obteniendo con ello una mirada integral del comportamiento individual e interrelacionado de las variables que lo componen, teniendo la ventaja de ofrecer una tipología de carácter empírica que describe y clasifica el fenómeno.

Como fuente principal de datos, mantenemos el reporte individual del adolescente y joven, el cual se utiliza permanentemente en la intervención dentro de los programas de Justicia Juvenil y específicamente, en el desarrollo de la Profundización Diagnóstica, clave para definir los objetivos a trabajar. Este reporte -que opera a través del profesional a cargo del caso, complementado y potenciado en las reuniones de equipo para el análisis a través de dinámica colectiva- consolida la mirada de la situación descrita por el instrumento.

Entre las posibilidades que ofrece el SIED-AJ, aparte de su gestión y aplicación sencilla, es que permite presentar gráficamente sus resultados. Ello permite sintetizar el comportamiento de las variables expresadas en el índice final, las cuales ubican la situación de cada caso dentro de una estructura de dimensiones e indicadores. La escala son instrumentos estandarizados que permiten expresar, en forma numérica, la posición relativa de un objeto en un continuo (Campbell, 1962).

Los indicadores del SIED-AJ están basados en diferentes tipos de escalas. A través del uso del juicio profesional y mediante un fichero que estandariza la aplicación, el profesional evalúa la complejidad de la situación que presenta el adolescente o joven al inicio del proceso (evaluación ex ante), durante el proceso (ex dure) y en la fase de término (evaluación ex post).

Se cuenta con tres tipos de indicadores:

- indicadores en formato de escala Likert (Likert, 1932), que oscila entre 1 y 5 puntos. En este caso un 1 indica un menor nivel de complejidad mientras que el 5 indica un nivel de mayor de complejidad.

- Escalas en las que se puede asignar una puntuación que considera los ejes centrales 1-3-5. En este caso también un 1 indica un menor nivel de complejidad mientras que el 5 indica un nivel de mayor de complejidad. 
- Indicadores con categorías dicotómicas lo observado.

En algunos casos se puede asignar un 0 , cuando el indicador no corresponde. Esto lo excluye de la medición.

\section{Elementos que componen la evaluación}

Como resultado de la evidencia teórica y empírica se diseñaron y definieron operacionalmente variables, asignándole una jerarquía que compone, a través de un peso específico, el índice final de complejidad.

- Historia infraccionaria: La historia de involucración en el tema infraccionario, su inicio y patrones básicos de conducta que quedan expuestos en cada una de las dimensiones definidas. Refieren los antecedentes que presenta el o la joven en relación con el contacto con el sistema judicial y el involucramiento anterior en la infracción de ley (Ref. Conceptual: meta análisis desarrollado por Zamble y Quinsey (1997).

- Aspectos personales: Se encuentran expuestos en cuatro dimensiones básicas que recogen una primera impresión en torno a los aspectos que componen la identidad personal, las características o rasgos de personalidad: agresividad, impulsividad, empatía y resolución de conflictos. Indican antecedentes respecto a las características individuales y comportamentales en cuanto a las habilidades y estrategias de autocontrol, y las que son favorables a la violencia (Ref. Conceptual: meta análisis desarrollado por Zamble y Quinsey (1997)).

- Motivación al cambio: Esta tercera variable refiere a la capacidad de resistencia y persistencia en el discurso sobre el tema infraccional. Es un elemento que resulta muy significativo al momento de ver cuán compleja es la situación que se aborda. Así, por ejemplo, los discursos cerrados y anclados en la identidad delictual son muy complejos al momento de definir algún cambio conductual. Por ello indica la disposición explicitada de enfrentar un proceso de cambio de cometer conductas infractoras (Ref. conceptual; CESC: dimensiones críticas de la intervención en reinserción y Guía de Evaluación SENAME, 2011).

- Consumo de sustancia: Para esta variable no existe evidencia concluyente que establezca una relación de causalidad entre la infracción de ley y el uso de sustancias, en tanto la evidencia establece una incidencia al momento de la infracción, sin que por ello dé cuenta de causalidad entre una y otra. El consumo de sustancia indica la relación, para efectos del diseño de complejidad en cuanto al patrón de consumo y su potencial impacto en la infracción de la ley (Zamble y Quinsey. 1997).

- Aspectos familiares: La familia es un referente significativo en la niñez del adolescente o joven. En la adolescencia puede convertirse en un contenedor o favorecedor de situaciones que lo pongan en riesgo. Las dinámicas familiares constituyen la herencia cultural y éstas permiten naturalizar conductas de espacios de socialización primaria. Un caso especial es la naturalización de dinámica disruptiva. Esta variable observa las dinámicas que refieren los antecedentes del grupo de socialización primaria (familiar cercano o significativo presente en el genograma) (Ref. Conceptual: metaanálisis desarrollado por Zamble y Quinsey (1997)).

- Grupo de Pares: Desde la sicología del desarrollo, en el período de la adolescencia cobra mayor importancia el grupo de pares en el proceso de socialización y de reforzamiento y modificación de dinámicas comportamentales o sociales. La posibilidad de considerar estas variables al momento de evaluar la complejidad insuma una dimensión difícil de sondear y, por tanto, de intervenir. No obstante, ofrece una mirada más integral para el reconocimiento de la situación en que se encuentra y percibe el o la adolescente o joven. La variable queda definida como los antecedentes que relacionan dinámicas del contexto territorial y grupo de pares con involucramiento en la infracción de la ley (Ref. Conceptual: meta análisis desarrollado por Zamble y Quinsey (1997), Matza (1981), Sutherland (1939)).

- Vinculación con el Entorno Significativo e Inserción Socioinstitucional: La finalidad de la sanción en su artículo 20 de la Ley 20.084 (Ley de Responsabilidad Penal Adolescente) define los propósitos de responsabilización por el hecho cometido y de promover la reintegración a la sociedad como principales objetivos en su ejecución. Por ello, antecedentes como el ámbito escolar, la inserción laboral son relevantes al momento de definir las estrategias que faciliten el cumplimiento de los objetivos de la intervención. En tanto, la experiencia de paso por el sistema de protección pone un antecedente en relación con la experiencia de vulneración que ha tenido el o la joven. Por ello, la definición operacional se encuentra relacionada con la obtención de antecedentes sobre la experiencia en términos de integración o reparación en tres ámbitos; edu- 
cativo, laboral y en relación con la intervención anterior como niño vulnerado (Ref. Conceptual: meta análisis desarrollado por Zamble y Quinsey (1997)).
- Salud Mental: La presencia del tema de Salud Mental modifica de manera transversal todas las otras variables evaluadas, alterando cualquiera de las condiciones descritas anteriormente, por lo que

FIGURA 1

EJEMPLO DE GESTIÓN DEL INSTRUMENTO (1A VARIABLE) Y CONTRASTE GRÁFICO DE EVALUACIÓN DE INICIO Y DE EGRESO

\begin{tabular}{|c|c|c|c|c|c|}
\hline Variable & $\%$ & Dimensión & & Categoría & Pts. \\
\hline \multirow{13}{*}{$\begin{array}{l}\text { Trayectoria y } \\
\text { aspectos } \\
\text { infraccionales }\end{array}$} & \multirow{14}{*}{0,855} & Edad de inicio & 5 & Tramos & 5 \\
\hline & & \multirow{5}{*}{ Delitos y habitualidad } & \multirow{5}{*}{2,6} & Delitos anteriores & 5 \\
\hline & & & & Habitualidad & 1 \\
\hline & & & & Delito al ingreso & 2 \\
\hline & & & & Delito más recurrente & 2 \\
\hline & & & & Delito más grave & 3 \\
\hline & & & & & \\
\hline & & \multirow{3}{*}{ Experiencia de prisionización } & \multirow{3}{*}{5} & Prisionización & 5 \\
\hline & & & & & \\
\hline & & & & Modalidad & 5 \\
\hline & & \multirow{3}{*}{ Sanción previa } & \multirow{3}{*}{4,5} & Sanción & 5 \\
\hline & & & & & \\
\hline & & & & Tipo & 4 \\
\hline & & Total & 4,275 & Alto & \\
\hline
\end{tabular}

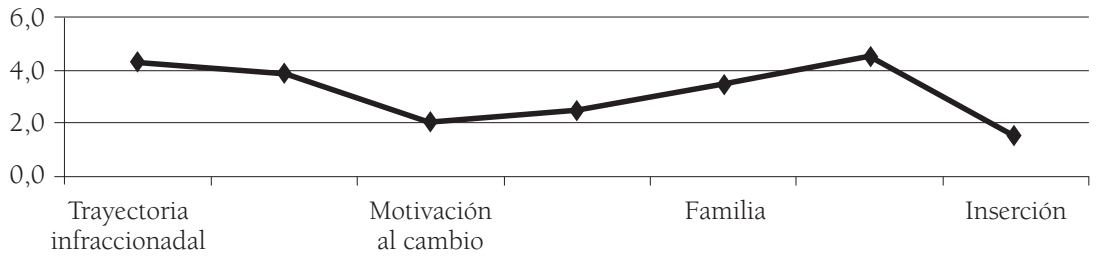

opera al final de la aplicación del instrumento con una corrección a las puntuaciones obtenidas, las que estarán afectas a la presencia o no de esta situación.

\section{Metodología}

Los datos utilizados para la medición y ponderación de las variables resultan de la aplicación de SIED-AJ a los adolescentes o jóvenes de los programas de sanciones de medio libre de Libertad Asistida y Libertad Asistida Especial, de las regiones de Iquique, Santiago, Rancagua, Linares y Cauquenes, Aysén y Magallanes, todos condenados por tribunales de garantía, tribunales de Juicio Oral en lo Penal o Corte de Apelaciones por infringir la ley.

La aplicación se realizó sobre una muestra de 176 casos en calidad de egresados por cumplimiento y remisión de la sanción de Libertad Asistida Especial, cuyos períodos se concentran entre el 1 de mayo de 2013 (fecha en que se comienza la sistematización de la información reportada respecto a dicho instrumentos por los equipos de Libertad Asistida Especial) y el 31 de marzo de 2014.

En este contexto se evidencia que todos los egresos de la muestra anteriormente descrita, que fluctúan en torno a una edad promedio de 20 años al término de la sanción, se sitúan en los rangos inferiores respecto del Nivel de Complejidad, siendo el 10\% de nuestros egresos de un nivel medio, el 50\% medio bajo y el $40 \%$ en la categoría bajo nivel de complejidad, lo que, según las impresiones preliminares de dichas cifras a nivel cualitativo, estimarían que aquellos casos que finalizan los procesos de intervención presentarían de manera progresiva una desvinculación de los grupos de pares en relación 
con la influenciabilidad que estos ejercen respecto a los procesos de toma de decisiones individuales, con experiencias de inserción ya sea en espacios de educación, formación y capacitación e inserción laboral (formal o informal) que han ampliado sus redes personales y sociales, lo que junto con un trabajo en el cual se han reforzado los factores protectores con los cuales ingresan, y tomando siempre en consideración las capacidades de respuesta, motivaciones e intereses, favorecerían la consolidación del desistimiento. Aquello se ve favorecido por el ciclo evolutivo que cursan los jóvenes, y en consistencia con una intervención que se diseñó precisamente para poder favorecer estos procesos de reinserción social, incluyendo los planes de inserción social, que, en la mayoría de los casos persigue la mantención y consolidación de los logros obtenidos.

Por último, cabe señalar que luego de ese primer análisis y entendiendo las propiedades psicométricas del instrumento se comenzó a aplicar ex dure, favoreciendo gestionar las intervenciones con mayor precisión y ajustando las variables según las necesidades de los adolescentes o jóvenes.

\section{Evaluación inicial de las propiedades psicométricas del instrumento}

Se hace referencia a la gráfica y su facilidad para ubicar los factores de riesgo y de protectores en cada caso, teniendo como referencia el medio de la escala (punto medio 3) para discernir entre uno y otro factor. En este caso, se puede apreciar un ejemplo en que las variables aparecen interrelacionadas y contrastadas entre la evaluación ex antes y en una segunda evaluación ex post realizada al egreso del proceso, observando las brechas que se producen entre las dos observaciones, exigiendo con ello, por parte del profesional a cargo del caso de una interpretación de los resultados, ya sea de aquellas modificaciones observables en cada una de las variables o su mantención, a modo de hipótesis explicativa del desarrollo del proceso.

\section{Pruebas de normalidad}

Las pruebas de normalidad que se aplicaron para ver el comportamiento del índice y su ponderación final, se realizó a través de los puntajes finales estandarizados, obtenidos en la primera etapa de aplicación del diseño ingresos y/o egresos entre enero de 2013 a julio de 2013, lo que significó un levantamiento de datos de 416 observaciones, entre ambos momentos de aplicación.

La distribución que presenta la tabla 2 responde a las diferentes puntuaciones para las medidas de ten- dencia central, las cuales se ubican, en el centro de la escala, en concordancia con las categorías definidas para la medición del índice final. Con media de 2,9, mediana 3 y moda 3 los estadígrafos se ubican prácticamente en el centro de la distribución, la cual presenta una dispersión de 0,93.

TABLA 1. TABLA DE ESTADÍGRAFOS

\begin{tabular}{|l|l|r|}
\hline \multirow{2}{*}{$\mathrm{N}$} & Válidos & 336 \\
\cline { 2 - 3 } & Perdidos & 1 \\
\hline Media & 2,91 \\
\hline Mediana & 3,00 \\
\hline Moda & 3 \\
\hline Desv. Tip. & 0,934 \\
\hline Varianza & 0,873 \\
\hline Asimetría & $-0,269$ \\
\hline Error tip. de asimetría & 0,133 \\
\hline Curtosis & $-0,473$ \\
\hline Error tip. de curtosis & 0,265 \\
\hline
\end{tabular}

Lo que podemos estimar es que las observaciones realizadas se asemejan a una distribución normal $\mathrm{N}$ $(0,1)$. Por otra parte, esta distribución consigna que se encuentra levemente cargada hacia la izquierda $(-0,26)$ de asimetría, lo que significa que hay más valores por debajo del promedio, y que presenta según la medida de curtosis menos datos en las colas de la distribución observada respecto de una distribución normal. En cuanto a la prueba específica de normalidad (tabla 3), esta presenta un nivel crítico superior a 0,05 en su significación, por lo cual se acepta la hipótesis de normalidad para esta distribución.

TABLA 2. PRUEBA DE NORMALIDAD

\begin{tabular}{|c|c|c|c|}
\hline \multicolumn{2}{|c|}{ Kolmogorov-Smirnov $^{\mathrm{a}}$} & \multicolumn{2}{c|}{ Shapiro-Wilk } \\
\hline Estadístico & Sig. & Estadístico & Sig. \\
\hline 0,049 & 0,020 & 0,986 & 0,000 \\
\hline
\end{tabular}

\section{Escala e índice final}

Para construir el índice final de complejidad se tiene como parámetro de referencia la propuesta de intervención licitada. En ella se encuentran estrategias que recogen las necesidades y características que exponen el número mayor de jóvenes atendidos, desde la implementación de la ley a la fecha, y ésta arroja lo que se considera al momento de elaborar los proyectos y se aplican a las herramientas de planificación como matrices lógicas y metodológicas, constituyendo la estructura gruesa de la propuesta licitada. 


\section{FIGURA 2. DISTRIBUCIÓN NORMAL DEL ÍNDICE}

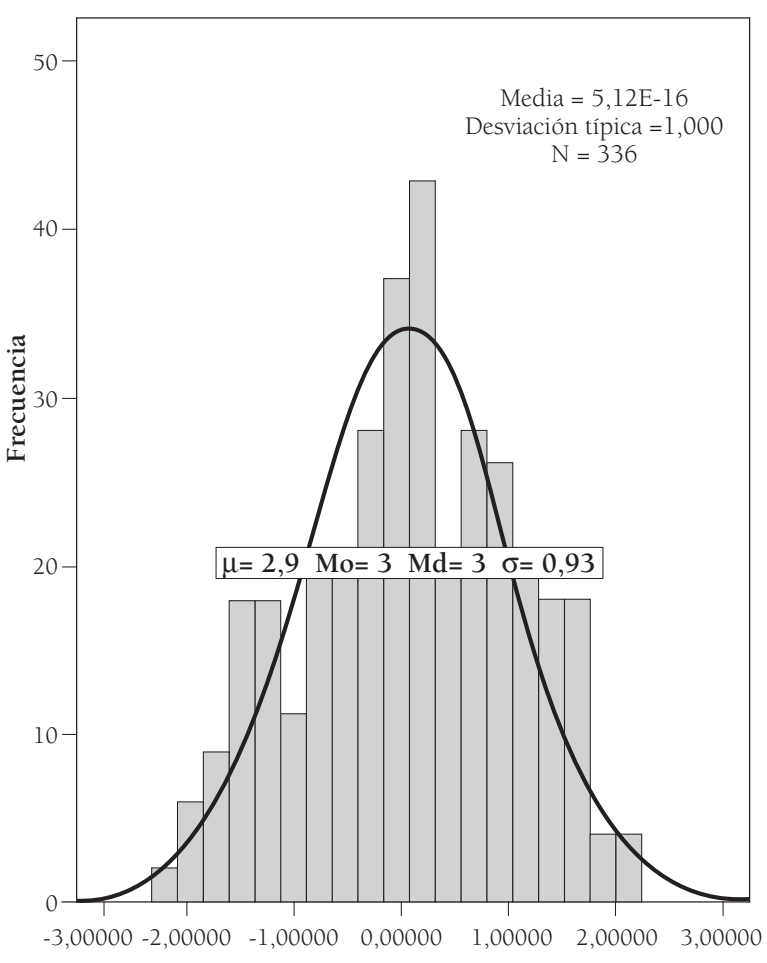

Puntua: FINAL PJORNADA

En términos matemáticos, la distribución normal representaría en el promedio y en el rango de 1 desviación estándar a ambos lados del estadígrafo, la mayor cantidad de casos $\mathrm{N}(0,1)$. El SIED-AJ como diseño de evaluación diferenciada se basa en puntajes que en cada uno de los ítems son asignados por la situación observada. Los puntajes límite (la amplitud total) recogidos desde la sumatoria de las escalas particulares que constituyen este índice van desde el parámetro menor, en escala conceptual $\operatorname{BAJO}(0,8)$, hasta el mayor puntaje que puede registrar como un nivel de complejidad ALTO $(4,97)$, y como representa la gráfica anterior, con una media poblacional de 2,9 y con un sigma o des- viación estándar poblacional de 0,93. Con estos parámetros es posible visualizar que los estadígrafos son coincidentes con los intervalos de puntajes definidos en la escala conceptualizada como BAJO, MEDIO/BAJO, MEDIO, MEDIO/ALTO, ALTO, encontrándose en este rango una alta probabilidad de ubicar un valor entre los puntajes que comprenden una desviación estándar en relación con la media $\mathrm{P}(1,97<\mathrm{x}<3,83)=68,3 \%$.

\section{Análisis de frabilidad}

Los resultados que aquí se exponen son producto del análisis de la aplicación del SIED-AJ como diseño de Evaluación Diferenciada en los programas de Justicia Juvenil de la Corporación Opción, específicamente en los programas de Libertad Asistida y Programa de Libertad Asistida Especial, implementados en la Región de Tarapacá (1 programa), Región Metropolitana (5), Rancagua (2), Linares (1), Cauquenes (1), Aysén (1) y Punta Arenas (1), alcanzando a 310 aplicaciones de ingresos realizadas entre enero y julio de 2013, incluyendo también a los casos que se encuentran vigentes antes de ese período y que cuentan con más de 3 meses de ingreso.

\section{TABLA 4. CONFIABILIDAD}

\begin{tabular}{|c|c|c|}
\hline Alfa de Cronbach & $\begin{array}{c}\text { Alfa de Cronbach } \\
\text { basada en los } \\
\text { elementos } \\
\text { tipificados }\end{array}$ & N de elementos \\
\hline 0,904 & 0,908 & 39 \\
\hline
\end{tabular}

Según la prueba estadística de fiabilidad, los índices presentan un nivel favorable referido a la consistencia interna del instrumento sometido a la prueba. Es así como lo demuestra la tabla 4, que representa un valor del Alfa de Cronbach consignado como alto, asociado a 0,9 , por lo que el instrumento cuenta con consistencia interna y fiabilidad.

TABLA 3. PUNTAJES DEL ÍNDICE DE COMPLEJIDAD

\begin{tabular}{|c|c|c|c|c|}
\hline \multicolumn{2}{|c|}{ Escala índice jornada } & & & \\
\hline ALTO & MEDIO/ALTO & MEDIO & MEDIO/BAJO & BAJO \\
\hline 5 & 4 & 3 & 2 & 1 \\
\hline $4,97-4,136$ & $4,135-3,302$ & $3,301-2,468$ & $2,467-1,634$ & $1,633-0,8$ \\
\hline
\end{tabular}

\begin{tabular}{|c|c|c|}
\hline 3,83 & 2,9 & 1,97 \\
\hline & $\mu=2,9$ & $\Sigma=0,93$ \\
\hline
\end{tabular}


En relación con el análisis de dos mitades, el instrumento genera un Alfa de Cronbach de 0,8 para ambas partes, con un consistente Coeficiente de Spearman-Brown de 0,7, lo cual permite pronosticar el aumento de fiabilidad obtenida tras el incremento de la longitud del test, siempre y cuando los ítems añadidos sean paralelos a los ya existentes. Según una tercera prueba, el Modelo de
Guttman, método basado en la reducción proporcional del error, indica que el error de predicción de una categoría de la variable pertenezca o se clasifique, considerando una variable seleccionada, teniendo en cuenta esa categoría, en cada categoría de una segunda variable. En este aspecto, los coeficientes definidos no bajan del 0,7 , siendo un valor de fiabilidad importante.

\section{FIGURA 3. ANÁLISIS DE DOS MITADES}

\begin{tabular}{|c|c|c|c|c|}
\hline & Media & Varianza & $\begin{array}{c}\text { Des- } \\
\text { viación } \\
\text { típica }\end{array}$ & $\begin{array}{c}\mathrm{N}^{\circ} \text { de } \\
\text { elementos }\end{array}$ \\
\hline Parte 1 & 54,84 & 199,475 & 14,124 & $20^{\mathrm{a}}$ \\
\hline Parte 2 & 51,46 & 227,306 & 15,077 & $19^{\mathrm{b}}$ \\
\hline $\begin{array}{c}\text { Ambas } \\
\text { partes }\end{array}$ & 106,29 & 699,522 & 26,448 & 39 \\
\hline
\end{tabular}

\begin{tabular}{|c|c|c|c|}
\hline \multirow{2}{*}{$\begin{array}{c}\text { Alfa de } \\
\text { Cronbach }\end{array}$} & \multirow{2}{*}{ Parte 1 } & Valor & 0,845 \\
\cline { 3 - 4 } & \multirow{2}{*}{ Parte 2 } & $\begin{array}{c}\mathrm{N}^{\circ} \mathrm{de} \\
\text { elementos }\end{array}$ & $20^{\mathrm{a}}$ \\
\cline { 3 - 4 } & & Valor & 0,852 \\
\cline { 3 - 4 } & & $\begin{array}{c}\mathrm{N}^{\circ} \text { de } \\
\text { elementos }\end{array}$ & $19^{\mathrm{b}}$ \\
\hline \multirow{2}{*}{$\begin{array}{c}\mathrm{N}^{\circ} \text { total de } \\
\text { elementos }\end{array}$} & 39 \\
\hline $\begin{array}{c}\text { Coeficiente } \\
\text { de Spearman- } \\
\text { Brow }\end{array}$ & $\begin{array}{c}\text { Correlación } \\
\text { entre formas }\end{array}$ & 0,640 \\
\hline & $\begin{array}{c}\text { Longitud } \\
\text { Igual }\end{array}$ & 0,781 \\
\hline & $\begin{array}{c}\text { Longitud } \\
\text { Desigual }\end{array}$ & 0,781 \\
\hline \multirow{2}{*}{$\begin{array}{c}\text { Dos mitades } \\
\text { de Guttman }\end{array}$} & 0,780 \\
\hline
\end{tabular}

Análisis de Fiabilidad Modelo Guttman

\begin{tabular}{|c|c|c|}
\hline \multirow{2}{*}{ Lambda } & 1 & 0,881 \\
\cline { 2 - 3 } & 2 & 0,910 \\
\cline { 2 - 3 } & 3 & 0,904 \\
\cline { 2 - 3 } & 4 & 0,780 \\
\cline { 2 - 3 } & 5 & 0,896 \\
\cline { 2 - 3 } & 6 & 0,947 \\
\hline & & 39 \\
\hline
\end{tabular}


h) Análisis específicos de fiabilidad

El análisis específico de confiabilidad pasa por la matriz de correlación global; que es contrastada a cada una de las categorías de SIED-AJ con la finalidad de dar consistencia y certeza metodológica. Como se observa en la tabla siguiente, las corre- laciones no son inferiores al 0,3 con lo cual para una nueva versión del diseño se requieren algunos ajustes, dado que estadísticamente no constituyen un aporte significativo a la consistencia del instrumento si se eliminaran.

TABLA 5. ANÁLISIS POR ÍTEMS

\begin{tabular}{|c|c|c|c|c|c|}
\hline \multicolumn{6}{|c|}{ EJEMPLO DE ANALISIS DE FIABILIDAD } \\
\hline & $\begin{array}{c}\text { Media de la } \\
\text { escala si se } \\
\text { elimina el } \\
\text { elemento }\end{array}$ & $\begin{array}{l}\text { Varianza de } \\
\text { la escala si } \\
\text { se elimina } \\
\text { el elemento }\end{array}$ & $\begin{array}{l}\text { Correlación } \\
\text { elemento total } \\
\text { corregida }\end{array}$ & $\begin{array}{c}\text { Correlación } \\
\text { múltiple al } \\
\text { cuadrado }\end{array}$ & $\begin{array}{c}\text { Alfa de } \\
\text { Cronbach si } \\
\text { se elimina el } \\
\text { elemento }\end{array}$ \\
\hline ACEPTACIÓN & 103,66 & 669,020 & 0,424 & 0,471 & 0,902 \\
\hline ACTITUD & 103,69 & 652,930 & 0,597 & 0,551 & 0,899 \\
\hline HOSTILIDAD & 103,41 & 661,054 & 0,504 & 0,525 & 0,901 \\
\hline HABITUALIDAD & 103,28 & 655,539 & 0,579 & 0,690 & 0,900 \\
\hline $\mathrm{EDAD} 1^{\circ} \mathrm{CON}$ & 102,93 & 654,798 & 0,535 & 0,551 & 0,900 \\
\hline RECO DAÑO & 103,73 & 652,647 & 0,525 & 0,578 & 0,900 \\
\hline TRATAMIENTO & 103,61 & 659,965 & 0,313 & 0,385 & 0,905 \\
\hline PASO SIST & 103,90 & 667,879 & 0,353 & 0,371 & 0,903 \\
\hline FAM C/ CONS & 103,51 & 664,746 & 0,395 & 0,405 & 0,902 \\
\hline ESTILO REL & 102,78 & 668,634 & 0,440 & 0,469 & 0,902 \\
\hline COMPROM & 104,12 & 666,730 & 0,440 & 0,468 & 0,902 \\
\hline CONO/TERR & 103,07 & 668,392 & 0,418 & 0,317 & 0,902 \\
\hline REL/TERRI & 102,76 & 654,872 & 0,626 & 0,601 & 0,899 \\
\hline REL/PARES & 102,72 & 651,257 & 0,627 & 0,626 & 0,899 \\
\hline \multicolumn{6}{|c|}{$\begin{array}{l}\text { ÍNDICE DE DISCRIMINACIÓN DE ÍTEMS>0,3 } \\
\text { (CORRELACIÓN DE ELEMENTOS) }\end{array}$} \\
\hline
\end{tabular}


Por otro lado, en la matriz de correlación interelementos, se constata que, si bien los ítems se encuentran relacionados, fueron seleccionados y revisados los que presentan una relación casi nula (cercanas al 0). Con ellos se realizarán ajustes al instrumento. Se expone como ejemplo la primera variable: Trayectoria Infraccional.

TABLA 6. EJEMPLO MATRIZ DE RELACIONES

\begin{tabular}{|c|c|c|c|c|c|c|c|c|c|c|}
\hline & Edad ini & $\begin{array}{l}\text { Del. An- } \\
\text { terior }\end{array}$ & $\begin{array}{l}\text { Habitua- } \\
\text { lidad }\end{array}$ & $\begin{array}{c}\text { Del. } \\
\text { Ingreso }\end{array}$ & $\begin{array}{l}\text { Del. + Re- } \\
\text { currente }\end{array}$ & $\begin{array}{l}\text { Del. + } \\
\text { Grave }\end{array}$ & Prisión & $\begin{array}{l}\text { Modali- } \\
\text { dad }\end{array}$ & Sanción & Tipo \\
\hline Edad ini & 1,000 & 0,466 & 0,320 & 0,012 & 0,236 & 0,212 & 0,204 & 0,240 & 0,254 & 0,261 \\
\hline $\begin{array}{l}\text { Del. } \\
\text { anterior }\end{array}$ & & 1,000 & 0,346 & $-0,048$ & 0,138 & 0,190 & 0,198 & 0,217 & 0,472 & 0,422 \\
\hline Habitualidad & & & 1,000 & 0,029 & 0,112 & 0,116 & 0,084 & 0,089 & 0,231 & 0,192 \\
\hline Del. ingreso & & & & 1,000 & 0,324 & 0,629 & 0,058 & 0,064 & $-0,147$ & $-0,084$ \\
\hline $\begin{array}{l}\text { Del. + } \\
\text { recurrente }\end{array}$ & & & & & 1,000 & 0,540 & 0,264 & 0,254 & 0,132 & 0,199 \\
\hline Del. + grave & & & & & & 1,000 & 0,147 & 0,146 & 0,053 & 0,082 \\
\hline Prisión & & & & & & & 1,000 & 0,928 & 0,452 & 0,522 \\
\hline Modalidad & & & & & & & & 1,000 & 0,459 & 0,577 \\
\hline Sanción & & & & & & & & & 1,000 & 0,832 \\
\hline Tiрo & & & & & & & & & & 1,000 \\
\hline Cuest 1 & & & & & & & & & & \\
\hline
\end{tabular}


El análisis de la matriz de correlaciones establece que en términos generales la primera variable (ASPECTOS INFRACCIONALES) presenta una in- cidencia mayor de casillas con relaciones muy bajas, casi nulas, corroborando estadísticamente que se comporta de manera independiente.

\begin{tabular}{|c|c|c|c|c|c|c|c|}
\hline \multirow[b]{2}{*}{ V1 } & Vl & V2 & V3 & V4 & V5 & V6 & V7 \\
\hline & $\begin{array}{r}7 \% \\
7 \\
100\end{array}$ & $\begin{array}{r}30 \% \\
24 \\
80\end{array}$ & $\begin{array}{r}13 \% \\
4 \\
30\end{array}$ & $\begin{array}{r}25 \% \\
10 \\
40\end{array}$ & $\begin{array}{r}45 \% \\
18 \\
40\end{array}$ & $\begin{array}{r}23 \% \\
7 \\
309\end{array}$ & $\begin{array}{r}42 \% \\
29 \\
70\end{array}$ \\
\hline \multirow{5}{*}{\multicolumn{2}{|c|}{ V2 }} & 0 & 0 & 0 & 3 & 0 & $\begin{array}{r}25 \% \\
14 \\
56\end{array}$ \\
\hline & & V3 & 0 & 1 & 0 & 0 & $\begin{array}{r}23 \% \\
5 \\
21\end{array}$ \\
\hline & & & V4 & 0 & 1 & 0 & $\begin{array}{r}18 \% \\
5 \\
28\end{array}$ \\
\hline & & & & V5 & 0 & 0 & $\begin{array}{r}7 \% \\
2 \\
28\end{array}$ \\
\hline & & & & & V6 & 0 & 2 \\
\hline \multicolumn{3}{|c|}{$\begin{array}{l}\text { \% casilla con correlación nula ( } 0) \\
\mathrm{F}(\mathrm{x}) \text { de casilla con correlación nula } \\
\text { Total de f(x) por casilla }\end{array}$} & & & & V7 & 1 \\
\hline
\end{tabular}

En menor magnitud también se aprecia una mayor cantidad de casillas con relaciones casi nulas en variable 7 (INSERCIÓN SOCIAL E INSTITUCIONALIZACIÓN), por lo que es necesario revisar y generar un ajuste de la categorías o ítems que la constituyen para mejorar estos indicadores en su aplicación.

\section{Conclusiones}

El SIED-AJ se orienta a responder la pregunta: ¿Cuál es la intervención adecuada para promover el desistimiento en un joven en particular? Este instrumento, desarrollado a partir de un acucioso trabajo que puso en conjunto la evidencia comparada y nacional disponible con la experiencia recolectada por más de 20 años de trabajo, permite identificar niveles de complejidad para orientar de manera idónea una intervención que dé cuenta de las necesidades específicas de cada joven o adolescente en particu- lar, atendiendo prioritariamente aquellos ámbitos de mayor urgencia o que sean determinantes en el repertorio de conductas de riesgo.

Este trabajo reconoce la relevancia de la evidencia local y capitaliza la experiencia de los propios equipos, dando espacio así a la inclusión del juicio experto no sólo en la aplicación, sino también en el diseño de un instrumento estandarizado de evaluación, que cumple con altos estándares en cuanto a su validación estadística.

En segundo lugar, el instrumento se basa en la idea de complejidad, lo cual da cuenta de que el proceso de desistimiento es paulatino, difícil y que incluye elementos tales como la madurez psicosocial, los lazos interpersonales, el contexto social y la motivación al cambio. Esto abre el camino para implementar intervenciones que recojan las narrativas y experiencias de los propios jóvenes, que rescaten sus potencialidades y que sean consistentes con sus 
intereses. Lo anterior es clave, al incorporar el hecho, muchas veces negado, de que la infracción de la ley para los jóvenes va más allá de la subsistencia económica, y que se relaciona con posibilidades de ejercer control, asegurar un sentido de identidad y tener una autopercepción de movilidad social.

En este sentido creemos que SIED-AJ incorpora elementos novedosos en el ámbito del diagnóstico a partir de sus propiedades psicométricas de evaluación que nos brindan resultados al ingreso, durante el proceso y al egreso de los adolescentes o jóvenes, permitiendo comparar las aplicaciones con la finalidad de gestionar intervenciones más adecuadas y atingentes.

Por último, creemos necesario seguir generando procesos de validez que nos permitan continuar y profundizar en todas las posibilidades que nos entrega SIED-AJ como es su aplicación en población femenina, cuestión no presente en otros instrumentos y que se releva toda vez que no hay respuestas publicas especializadas que incorporen la perspectiva de género.

\section{Bibliografía}

BIBLIOTECA DEL CONGRESO NACIONAL DE CHILE

(2005). Ley de Responsabilidad Penal Adolescente (ley 20.084). Disponible en http://www.leychile.cl/Consulta/listaresultadosimple?cadena=ley+de+responsabilida d+penal+adolescente (Extraído el 20 de abril de 2011).

BONTA, J Y ANDREWS D. A. (2006). Riesgo-NecesidadResponsividad. Modelo de evaluación y rehabilitación de infractores. Public Safety Canada. Carleton University.

BRONFENBRENNER The Ecology of Human Development: Experiments by Nature and Design, Harvard University Press, Cambridge MA, 1979.

BUSTILLOS, G., VARGAS, L. (2001). Técnicas participativas para la educación popular. Guadalajara, Jal.: IMDEC.

CONVENCIÓN INTERNACIONAL SOBRE LOS DERECHOS DEL NIÑO (CIDN) (1990). Disponible en http://www2.ohchr.org/spanish/law/crc.htm (Acceso 20 de abril de 2011).

CORPORACIÓN OPCIÓN, UNICEF (2009). Conoce tus derechos. Manual sobre la Ley de Responsabilidad Penal de Adolescentes. Santiago, Chile.

CONFLICT RESOLUTION NETWORK (2003). Las doce destrezas de resolución de conflictos y el juego de resolución de conflictos: Una alternativa al debate tradicional. Traducido por Morale, I y De la Paz, A. Disponible en http://www.conversacionesnuevas.net/ doce_destrezas.pdf (Acceso marzo 2015)
DROPPELMANN, C. (2009). Estudio sobre modelos de intervención y administración de centros privativos de libertad en la reforma penal adolescente, informe final. Capítulo 2: Evaluación, clasificación y manejo de casos en los centros privativos de libertad para jóvenes. Santiago. Chile. Fundación Paz Ciudadana, Chile.

DROPPELMANN, C Y HEIN, A (2010). Manual para la aplicación y corrección del instrumento para la detección de problemas propios de la adolescencia asociados al uso de drogas; POSIT, Núcleo Científico Mileniode análisis socioeconómicos sobre uso y abuso de drogas, Instituto de Sociología PUC, Chile.

ESPINOZA, O. ET AL., (2012). Estudio modelo y medición de la reincidencia de los adolescentes y jóvenes infractores de la ley penal. Centro de Estudios en Seguridad Ciudadana, Instituto de Asuntos Públicos, Universidad de Chile.

GREGORY, C. (1967). The Management of intelligence: Scientific Problem Solving and Creativity. McGrawHill. USA.

NACIONES UNIDAS (1948). Declaración Universal de los Derechos Humanos. Adoptadas y proclamadas por la Asamblea General.

MARSHALL, T. (1999). Restorative Justice: An Overview. London: Home Office Research Development and Statistics Directorate. Traducción propia, p. 5.

MCNEILL, F. (2012) Paradigma del desistimiento para la gestión de delincuentes. DOCUMENTO DE TRABAJO $\mathrm{N}^{\circ}$ 27. Universities of Glasgow and Strathclyde, UK.

MOLINÉ, J. Y OLIVÉ, J. (2010). El proceso de desistimiento de las personas encarceladas. Obstáculos y apoyos. Documentos de trabajo. Ambit social i criminologic. Departamento de Justicia Cataluña, España.

SENAME (2012). Orientaciones Técnicas para la intervención. Programa Libertad Asistida Especial. Documento de Trabajo, Ministerio de Justicia, Chile.

SILVA, D. (2003). La acción educativa liberadora en contextos de control social: Buscando estrategias de disminución de la vulnerabilidad al sistema punitivo y de reducción de la violencia de las respuestas penales.

TORRICO E. Y SANTÍN C. (2002). El modelo ecológico de Bronfrenbrenner como marco teórico de la Psicología, vol. 18, no 1, pp. 45-59. Servicio de Publicaciones de la Universidad de Murcia, España.

VÁZQUEZ ROSSONI, OSVALDO (2003), Responsabilidad Penal de Adolescentes, Corporación Opción, Santiago. 